\title{
IMPROVEMENT OF BIOGAS PRODUCTION IN ANAEROBIC DIGESTION PROCESS
}

\author{
Abdelhani Chaabna, Samia Semcheddine
}

\begin{abstract}
The production of biogas enables environmental preservation and sustainable development of rural areas and landlocked regions, as well as diversification of renewable energy resources. This paper is a contribution to improving the production of biogas by Sliding Mode Control (SMC).

In the literature there are many models describing the behaviour of reactions during anaerobic digestion and used for control design. The AM2 model is one of the simplest models and can be exploited easily for the control design purposes. In this paper, the reduced model AM2 was exploited to develop and testing by simulations the robust control law SMC.

The results obtained have proved the effectiveness of the control method proposed in this paper. A study of the robustness for monitoring and disturbances rejection demonstrated the great interest of this method, which is a nonlinear technique and gives very good results in terms of robustness but it presents the problem of chattering. In practice, the chattering of the control action can cause premature wear of the actuators or parts of the system due to heavy oscillations. The chattering phenomenon is caused by the discontinuous term which appears in the control signal. This paper presents a solution to the chattering problem by replacing the discontinuous term with a continuous one. Different Simulations and comparisons are presented and interpreted with satisfactory results.
\end{abstract}

Keywords: Anaerobic digestion, SMC, chattering, robustness, nonlinear control

\section{INTRODUCTION}

Anaerobic digestion has many advantages because it allows to obtain renewable energy that does not depend on climatic conditions but on the contrary it is available during the 4 seasons of the year. It is available everywhere since the organic waste that makes it possible to obtain this renewable energy is available everywhere. But this energy must be exploitable in areas of the agrofood industry. On farms where animal waste can be used and in remote and isolated areas when it is difficult and expensive to make the necessary installations for natural gas.

The downside of these processes is that they are known to become easily unstable under variations of operating conditions [1]. Then a feedback control loop can ensure stability and improve performance [2].

Therefore, a reliable dynamic model of the process is required for the design of such monitoring and control algorithms.

The representation of the process was then improved by considering three stages: solubilization of organics, acidogenesis, and methanogenesis [3].

Many mathematical models of these processes in continuously stirred tank reactor (CSTR) are presented by nonlinear ordinary differential equations.

The five orders $\mathrm{AD}$ model may the realistic basis for theoretical control algorithm design [4], [5] where a synergetic procedure and an extremum-seeking control design for anaerobic digestion processes are employed to derive the control output. They can achieve a high robustness, fast convergence, low steady state error, and globally uniformly asymptotic stability.
Mosey [6] introduced a four-population model with two acidogenesis reactions and two methanization reactions which also emphasizes the role of hydrogen. These main modelling studies have then been extended and detailed by other authors in order to get Identifiable, we assume that the bacterial populations can be divided into two main groups of homogenous characteristics, and that the anaerobic digestion can be described by a two-stage process. in the first step (acidogenesis), the acidogenic bacteria $\left(\mathrm{X}_{1}\right)$ consume the organic substrate $\left(\mathrm{S}_{1}\right)$ and produce $\mathrm{CO}_{2}$ and volatile fatty acids $\left(\mathrm{S}_{2}\right)$, the population of methanogenic bacteria $\left(X_{2}\right)$ uses, in a second step, the volatile fatty acids as substrate for growth and produce $\mathrm{CO}_{2}$ and methane.

The rest of the paper is organized as follows: First, process description is briefly recalled followed by a brief Sliding Mode Control (SMC) section. Simulation and results are then developed. The proposed SMC with its results of the simulation are presented and discussed in subsequent sections.

\section{OVERALL MODEL CONFIGURATION}

The AM2 model $[7,8]$ takes place in 2 stages thus describing the acidification and methanization process.

This model was selected among many others in this paper for many reasons:

1) It is a simple model (reflects two phases of the process) therefore easily usable in control.

2) A parameter $\alpha$ was introduced (eq1) in order to model biomass retention $\alpha=0$ corresponds to an ideal fixed-bed reaction while $\alpha=1$ corresponds to an ideal reactor with no biomass retention system. 
3) It takes into account the inhibition phenomenon thanks to growth rate $\mu_{2}$ modeled by Haldane (eq3).

4) one has the relations which binds the ADM1 and the AM2 in the reference [8] and consequently one can apply the results obtained in this paper on the ADM1.

The mathematical model is expressed as a system of first order differential equations of the form:

$$
\begin{aligned}
& \frac{d X_{1}}{d t}=\left(\mu_{1}\left(S_{1}\right)-\alpha D\right) X_{1} \\
& \frac{d X_{2}}{d t}=\left(\mu_{2}\left(S_{2}\right)-\alpha D\right) X_{2} \\
& \frac{d S_{1}}{d t}=D\left(S_{1 i n}-S_{1}\right)-k_{1} \mu_{1}\left(S_{1}\right) X_{1} \\
& \frac{d S_{2}}{d t}=D\left(S_{2 i n}-S_{2}\right)+k_{2} \mu_{1}\left(S_{1}\right) X_{1}-k_{3} \mu_{2}\left(S_{2}\right) X_{2}
\end{aligned}
$$

The mathematical model based on the laws of growth involves the following dynamic variables.

$X_{1}$ and $X_{2}$ are respectively the concentration of the acidogenic bacteria population and the concentration of the methanogenic bacteria population.

$S_{1}$ and $S_{2}$, are respectively the concentration of the organic substrate and the concentration the volatile fatty acids (VFA) in the bioreactor. D is the dilution rate.

$\mu_{1}$ and $\mu_{2}\left(\mathrm{~d}^{-1}\right)$ represent the specific growth rates of acidogenesis and methanization, respectively.

We consider Monod-type kinetics for the grwth of acidogenic bacteria that is:

$$
\mu_{1}\left(S_{1}\right)=\frac{m_{1} S_{1}}{S_{1}+K_{1}}
$$

Where $m_{1}$ is the maximum bacterial growth rate, and $K_{1}$ is the half-saturation constant associated with the substrate $S_{1}$.

In order to emphasize the possible VFA accumulation, Haladane kinetics for the methanogenesis is considered

$$
\mu_{2}\left(S_{2}\right)=\frac{m_{2} S_{2}}{K_{2}+S_{2}+S_{2}^{2} / K_{i}}
$$

Where $m_{2}$ is the maximum bacterial growth rate without inhibition, and $\mathrm{K}_{2}$ and $\mathrm{K}_{\mathrm{i}}$ are the saturation and inhibition constants associated with the substrate $\mathrm{S}_{2}$ respectively.

Moreover, the methane flow rate, which is the expected product, depends directly on the growth of the methanogenic bacterial population $\mathrm{X}_{2}$, according to the relationship:

$$
\mathrm{Q}=\mathrm{k}_{6} \mu_{2} \mathrm{X}_{2}
$$

\section{PRINCIPLE AND CALCULATION OF THE SMC CONTROL LAW}

The sliding mode control has experienced a considerable development $[9,10]$, this is mainly due to the property of rapid convergence, as well as the great robustness with respect to modeling errors and certain types of external disturbances.

All these positive aspects should not, however, mask certain drawbacks. The sliding mode controls operate discontinuously.

The discontinuities of the control algorithm occur directly on the actuator. If this organ is not designed for this type of solicitation, this risks leading to its premature aging and the system will be subjected at all times to a high control in order to ensure its convergence to the desired state.

The sliding surface is considered to be the error given by the relation where $\mathrm{e}(\mathrm{t})$ is the error between desired methane flow rate and real methane flow rate " $Q d$ " and " $Q$ ",

$$
S=e=Q d-Q
$$

Typically, SMC includes two parts; equivalent and discontinuous parts, as given in $\mathrm{Eq}(6)$ :

$$
D(t)=D_{e q}(t)+D_{\text {dis }}(t)
$$

To get the equivalent control signal, $\mathrm{Eq}(5)$ must be derived and made equal to zero as follows:

$$
\dot{S}=-k_{6} \dot{\mu} 2 X_{2}+-k_{6} \mu 2^{2} X_{2}+k_{6} \mu 2 \alpha D X_{2}=0
$$

Hence, the expression of $D_{e q}$ is deduced as: 


$$
D_{e q}=\frac{m_{2}\left(K_{2}-\frac{S_{2}^{2}}{K_{i}}\right)\left(k_{2} \mu_{1}\left(S_{1}\right) X_{1}-k_{3} \mu 2\left(S_{2}\right) X_{2}\right)-\left(K_{2}+S_{2}+\left(S_{2}^{2}\right) / K_{i}\right)^{2} \mu 2^{2}}{-m_{2}\left(K_{2}-\frac{S_{2}^{2}}{K_{i}}\right)\left(S_{2 i n}-S_{2}\right)+\left(K_{2}+S_{2}+\left(S_{2}^{2}\right) / K_{i}\right)^{2} \mu 2 \alpha}
$$

We add to this term a discontinuous term and then the control law becomes:

$$
D=\frac{m_{2}\left(K_{2}-\frac{S_{2}^{2}}{K_{i}}\right)\left(k_{2} \mu_{1}\left(S_{1}\right) X_{1}-k_{3} \mu 2\left(S_{2}\right) X_{2}\right)-\left(K_{2}+S_{2}+\left(S_{2}^{2}\right) / K_{i}\right)^{2} \mu 2^{2}}{-m_{2}\left(K_{2}-\frac{S_{2}^{2}}{K_{i}}\right)\left(S_{2 i n}-S_{2}\right)+\left(K_{2}+S_{2}+\left(S_{2}^{2}\right) / K_{i}\right)^{2} \mu 2 \alpha}+\lambda \operatorname{sign}(S)
$$

\section{PROPOSED SMC CONTROL}

Many solutions have been proposed with the aim of reducing or eliminating this phenomenon. These methods reduce the robustness of the control law.

They are parameterized by a positive constant $\varepsilon$ adjusted to have a good compromise between reduction of chattering and conservation of robustness.
In the method presented here, the smaller $\varepsilon$ more the approximation tends towards the sign function and therefore the better the robustness to the detriment of the reduction in chattering.

The approximation presented in this paper consists in replacing the sign function by the continuous function $\frac{S}{|S|+\varepsilon}$ proposed by Ambrosino et al [11].This function is shown in Fig.1.

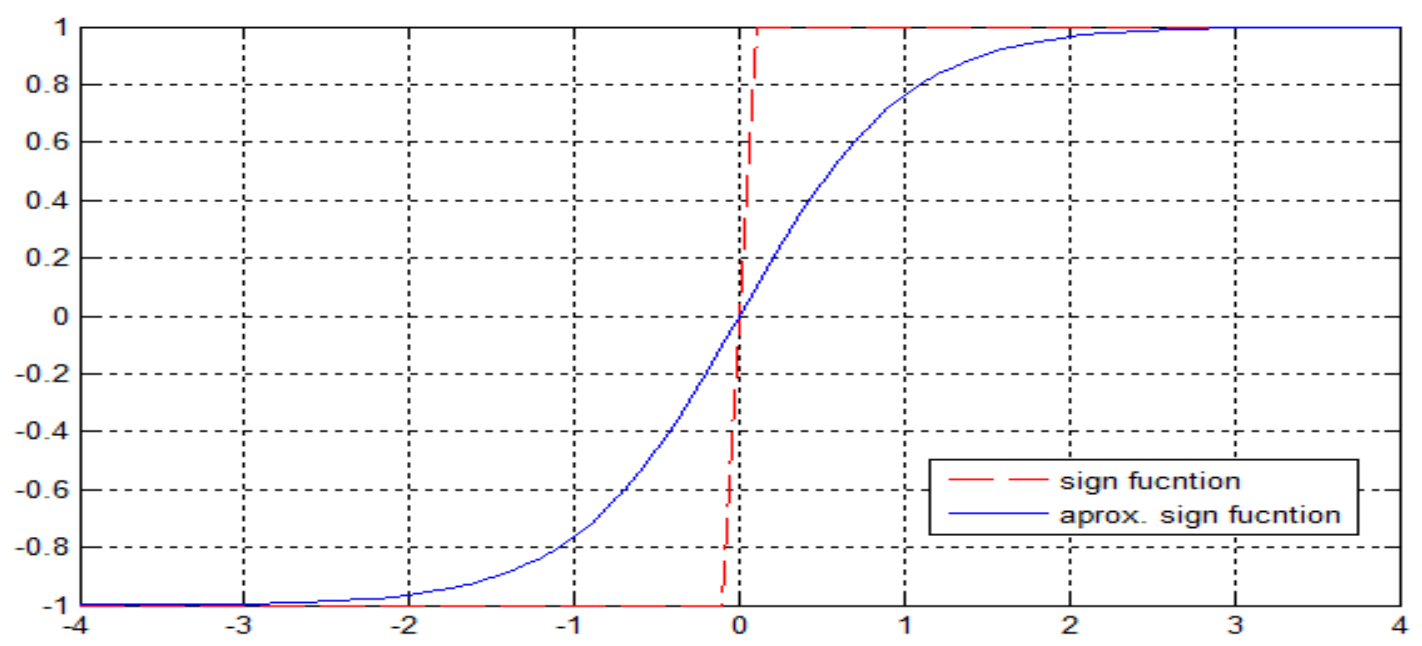

Fig. 1. Continuous approximation of the signum function

Which gives for D the relation (10):

$$
D=\frac{m_{2}\left(K_{*_{2}}-\frac{S_{2}^{2}}{K_{i}}\right)\left(k_{2} \mu_{1}\left(S_{1}\right) X_{1}-k_{3} \mu_{2}\left(S_{2}\right) X_{2}\right)-\left(K_{2}+S_{2}+\frac{S_{2}^{2}}{K_{i}}\right)^{2} \mu_{2}^{2}}{-m_{2}\left(K_{*_{2}}-\frac{S_{2}^{2}}{K_{i}}\right)\left(S_{2 i n}-S_{2}\right)+\left(K_{2}+S_{2}+\frac{S_{2}^{2}}{K_{i}}\right)^{2} \mu_{2} \alpha}+\lambda \frac{S}{|S|+\varepsilon}
$$




\section{SIMULATION RESULTS}

In order to confirm and compare the performance of the proposed SMC and classical SMC, numerical simulations are carried out using Matlab/Simulink environment. Parameters used in simulation are

$$
\begin{aligned}
& m_{1}=1.2, m_{2}=1.1, k_{1}=25, k_{2}=250, \\
& k_{3}=268, K_{i}=40, K_{1}=2, K_{2}=10, \\
& k_{6}=453 \alpha=0.5, S_{1 \text { in }}=32.0120, \lambda=0.2
\end{aligned}
$$

The initial conditions are given by

$$
\begin{aligned}
& S_{10}=0.5833, S_{20}=3.4068, \\
& X_{10}=1.1871, X_{20}=1.0645 .
\end{aligned}
$$

The studied system is tested under fast change $Q_{d}$ as shown in Fig. 3 with a disturbance on the $S_{2 \text { in }}$ input as shown in Fig. 2.

In order to properly test this nonlinear control law, we will insert a disturbance on the $S_{2 i n}$ input between times 100 days and 150 days (Fig. 2).

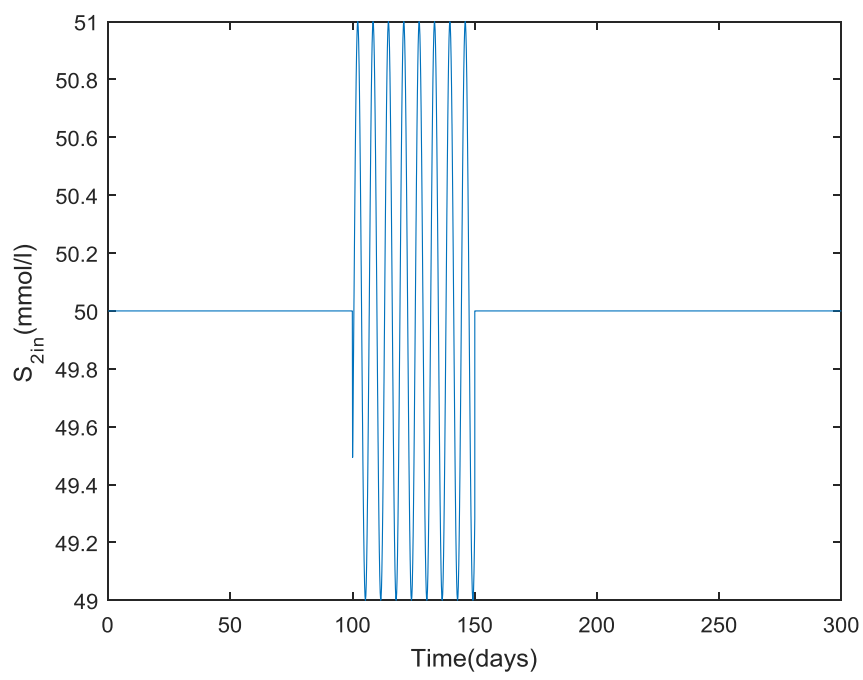

Fig. 2. Injected disturbance between 100 and 150 days

In the Fig. 3 obtained by simulation (Simulink) we can see the robustness of the control law suggested in this paper. In contrast, a high tracking performance was exhibited by using the classical SMC scheme. Also an instantaneous effect is displayed under desired methane flow rate changes and disturbance in $S_{2 i n}$ input without overshoots and with less fluctuation. Moreover, response time is significantly small.

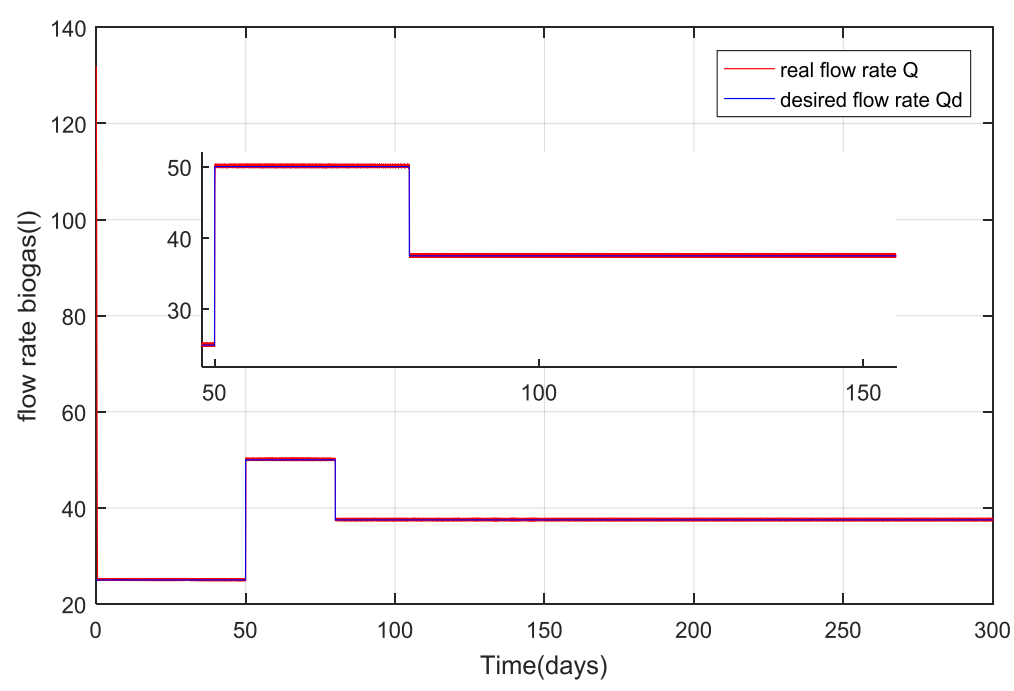

Fig. 3. Produced methane flow rate under classical SMC 
In Fig. 4 we observe that the control input $\mathrm{D}$ is always positive but it presents a lot of chattering.
After simulating, Figs. 5 and 6 show an important reduction of the chattering phenomenon.

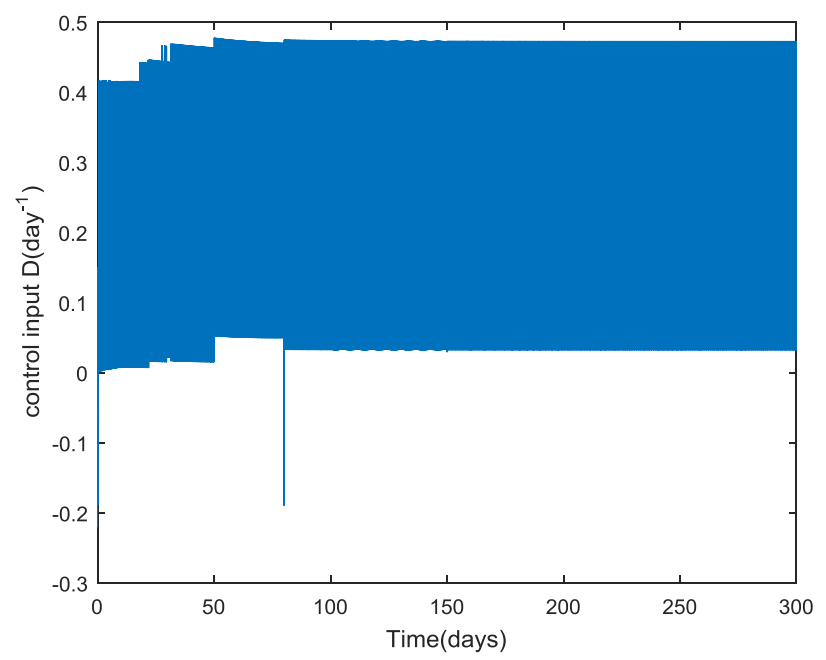

Fig. 4. Control input D under classical SMC

The obtained results shown in Figures 5 and 6 confirm the tracking superiority of the proposed control scheme compared to the conventional one under desired methane flow rate changes and disturbance in $S_{2 i n}$ input. It can be observed that by applying the proposed control scheme, methane flow rate is completely regulated to its reference with a reduction in the chatter

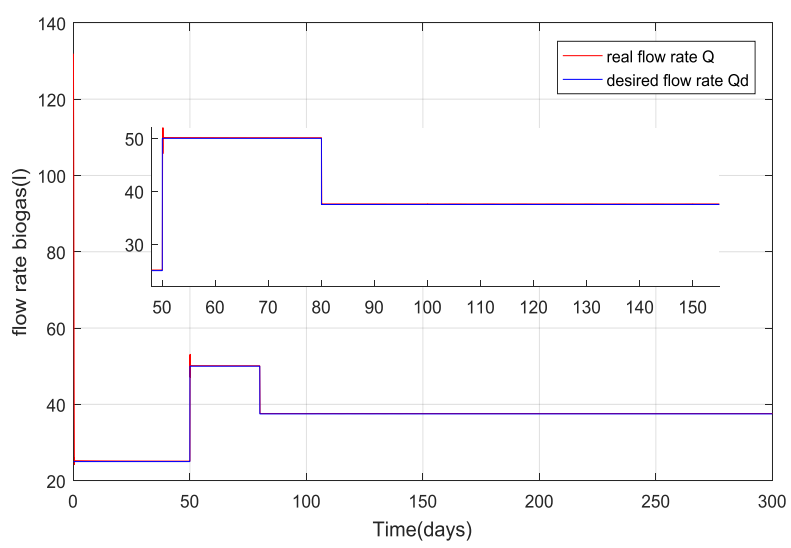

Fig. 5. Produced methane flow rate under proposed SMC.

\section{CONCLUSION}

A novel SMC method has been designed and detailed in this paper. The methane flow rate $\mathrm{Q}$ generated by SMC algorithm is accurately tracked through the desired. The performance of the proposed scheme has been tested through numerical simulations. Moreover, it was compared with the conventional SMC. The results obtained demonstrate that with the phenomenon. It is found that control input (D) exhibits important less fluctuation compared to the conventional control as shown in figure 6 and compared to Fig. 4.

It is worth mentioning that compared to the others methods, the proposed control demonstrates performance superiority in terms of fluctuation in steady state.

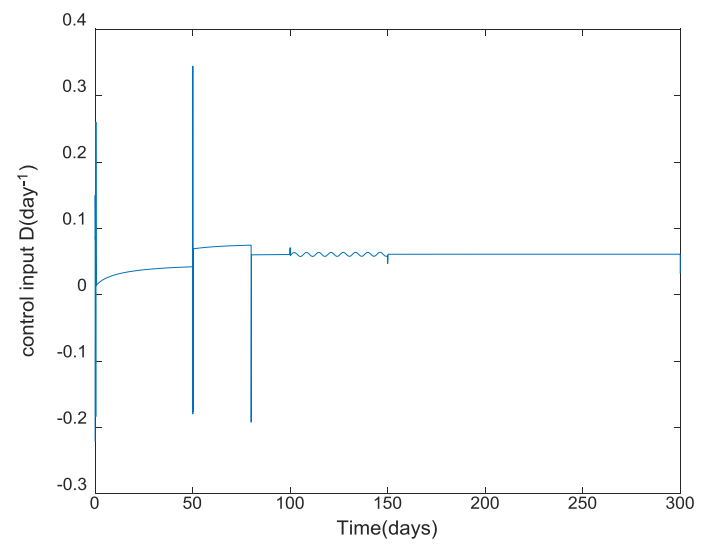

Fig. 6. Control input D under proposed SMC

proposed scheme D fluctuation (ripples) is minimized. Furthermore, tracking efficiency is enhanced.

Finally, the results obtained in this paper have been tested by simulation. The real anaerobic digestion process is very complex, we still have to test it on other models better descriptive of the process (three phase model) before testing it in practice. 


\section{REFERENCES}

1. J. J. Fripiat, M. Letellier, and P. Levitz, "Interaction of water with clay surfaces.," Philos. Trans. R. Soc. London, no. A311, pp. 287-299, 1984, doi: 10.1098/rsta.1984.0029.

2. D. Dochain, "Design of adaptive controllers for non-linear stirred tank bioreactors: extension to the MIMO situation," J. Process Control, vol. 1, no. 1, pp. 41-48, 1991, doi: 10.1016/0959-1524(91)87006-J.

3. D. T. Hill and C. L. Barth, "A Dynamic Model for Simulation of Animal Waste Digestion", J. Water Pollut. Control Fed., vol. 49, no. 10, pp. 2129-2143, Aug. 1977, Online. Available: http: //www.jstor.org/stable/25039421.

4. S. Semcheddine and H. Bouchareb, "Robust Control and State Estimation of a Three-Stage Anaerobic Digestion Process", Ecol. Eng. Environ. Prot., no. 2, pp. 29-38, 2019.

doi: 10.32006/eeep.2019.2.2938.

5. M. Hu, H. Wang, Y. Tian, N. Christov, and I. Simeonov, "On the Extremum-Seeking Control Design and Application for Anaerobic Digestion Processes", Ecol. Eng. Environ. Prot., no. 2, pp. 23 28, 2019, doi: 10.32006/eeep.2019.2.2328.

6. F. E. Mosey, "Mathematical Modelling of the Anaerobic Digestion Process: Regulatory Mechanisms for the Formation of Short-Chain Volatile Acids from
Glucose," Water Sci. Technol., vol. 15, no. 8-9, pp. 209-232, Aug. 1983, doi: 10.2166/wst.1983.0168.

7. O. Bernard, Z. Hadj-Sadok, D. Dochain, A. Genovesi, and J. P. Steyer, "Dynamical model development and parameter identification for an anaerobic wastewater treatment process.," Biotechnol. Bioeng., vol. 75, no. 4, pp. 424-438, Nov. 2001. doi: 10.1002/bit.10036.

8. S. Hassam, E. Ficara, A. Leva, and J. Harmand, "A generic and systematic procedure to derive a simplified model from the anaerobic digestion model No. 1 (ADM1)", Biochem. Eng. J., vol. 99, pp. 193203, Jul. 2015, doi: 10.1016/j.bej.2015.03.007.

9. I. Boiko, L. Fridman, A. Pisano, and E. Usai, "Analysis of chattering in systems with second-order sliding modes", IEEE Trans. Automat. Contr., vol. 52, no. 11, pp. 2085-2102, 2007.

doi: 10.1109/TAC.2007.908319

10. V. Utkin and H. Lee, "Chattering Problem in Sliding Mode Control Systems", in International Workshop on Variable Structure Systems, 2006. VSS'06., 2006, pp. 346-350.

doi: 10.1109/VSS.2006.1644542

11. G. Ambrosino, G. Celektano, and F. Garofalo, "Variable structure model reference adaptive control systems", Int. J. Control, vol. 39, no. 6, pp. 13391349, 1984, doi: 10.1080/00207178408933250.

\section{Abdelhani Chaabna, Samia Semcheddine}

LEPCI Laboratory, Faculty of Technology, Ferhat Abbas University, Setif1, Algeria;

e-mails: abdelhani.chaabna@univ-setif.dz, samia.semcheddine@univ-setif.dz 